\title{
A Coated Spherical Micro-Resonator for Measurement of Water Vapor Concentration at ppm Levels in Very Low Humidity Environments
}

\author{
Arun Mallik \\ Technological University Dublin, arun.mallik@tudublin.ie \\ Gerald Farrell \\ Technological University Dublin, gerald.farrell@tudublin.ie \\ Dejun Liu \\ Technological University Dublin, dejun.liu@tudublin.ie
}

See next page for additional authors

Follow this and additional works at: https://arrow.tudublin.ie/engschmanconn

Part of the Engineering Commons

\section{Recommended Citation Technology, IEEE. doi:10.1109/JLT.2018.2821272 \\ This Article is brought to you for free and open access by the School of Manufacturing and Design Engineering at ARROW@TU Dublin. It has been accepted for inclusion in Conference Papers by an authorized administrator of ARROW@TU Dublin. For more information, please contact arrow.admin@tudublin.ie, aisling.coyne@tudublin.ie,gerard.connolly@tudublin.ie.}

Mallik, A., Farrell, G. \& Liu, D. (2018). A Coated Spherical Micro-Resonator for Measurement of Water Vapor Concentration at ppm Levels in Very Low Humidity Environments. Journal of Lightwave

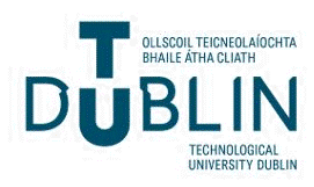




\section{Authors}

Arun Mallik, Gerald Farrell, Dejun Liu, Vishnu Kavungal, Qiang wu, and Yuliya Semenova

This article is available at ARROW@TU Dublin: https://arrow.tudublin.ie/engschmanconn/52 


\title{
A Coated Spherical Micro-Resonator for Measurement of Water Vapor Concentration at ppm Levels in Very Low Humidity Environments
}

\author{
Arun Kumar Mallik, Gerald Farrell, Dejun Liu, Vishnu Kavungal, Qiang Wu and Yuliya Semenova
}

\begin{abstract}
This paper presents a novel approach to measurements of low relative humidity $(\mathrm{RH})$ values based on the whispering gallery modes (WGMs) effect in a small silica microsphere coated with a thin layer of Agarose hydrogel. The light from a narrow-linewidth tunable laser with a few $\mathbf{G H z}$ tuning range is launched into an adiabatic tapered fiber, which excites WGMs in the Agarose layer via evanescent coupling. Adsorption and desorption of water vapor by the Agarose layer in response to changes in ambient humidity lead to changes in the layer's refractive index, which in turn leads to a spectral shift of the WGM resonances. We experimentally demonstrate the WGMs spectral shift over a range of RH values from $1 \% \mathrm{RH}$ to $25 \% \mathrm{RH}$. The proposed sensor displays linear response and is capable of measurement of low concentrations of water vapor (corresponding to about $11.7 \pm 0.32 \mathrm{ppm}$ water molecules in air). In addition, the proposed sensor offers the advantages of a very small form factor and good repeatability. Detailed studies of the sensor stability, cross sensitivity to temperature, response times and hysteresis are also presented.
\end{abstract}

Index Terms - Fiber optic sensor, micro-optical devices, optical microresonator, whispering gallery modes.

\section{INTRODUCTION}

$\mathrm{W}$ HISPERING gallery mode (WGM) optical microresonators are well known for their compactness, ultrahigh quality factors and small mode volumes. A large amount of research has been carried out in the field of physical, chemical, and biological quantities detection using such resonators [1-5]. WGMs are high angular momentum modes which can be excited by trapping light inside a dielectric structure with circular symmetry, such as a microsphere, by repeated (near-) total internal reflections. Resonances occur when light retains the same phase after each cycle of propagation. The spectral positions of the WGM resonances are strongly dependent on the geometry of the dielectric

This work was supported in part by the Dublin Institute of Technology and the Fiosraigh Dean of Graduate Students scholarship and by the Open Fund of IPOC, Beijing University of Posts and Telecommunications.

Arun Kumar Mallik, Gerald Farrell, Dejun Liu, Vishnu Kavungal and Yuliya Semenova (corresponding author) are with Photonics Research Centre, Dublin Institute of Technology, Kevin St, Dublin 8, Ireland (e-mail: yuliya.semenova@dit.ie).

Qiang Wu is with the Department of Mathematics, Physics and Electrical Engineering, Northumbria University, Newcastle Upon Tyne, NE1 8ST, UK. resonator (diameter, sphericity), the optical properties of the resonator material and on the refractive index (RI) of the surrounding the resonator medium. This makes such a device suitable for sensing of the surrounding RI or various chemical or biological species if a suitable functional coating is applied to its surface. For example, polymer-coated spherical microresonators have been previously developed for chemical vapor sensing [6], where a change in the polymer RI was induced by adsorption of ammonia molecules from the local environment. There are different approaches to fabrication of dielectric microresonators. Silica microspheres are easily fabricated by surface tension during melting of a silica fiber tip, resulting in a clean, smooth surface with low optical loss and negligible scattering.

Relative humidity $(\mathrm{RH})$ is one of important environmental conditions usually specified for cleanroom operations in the semiconductor, aerospace, food, and pharmaceutical industries. Humidity measurement in industry is often critical because incorrect humidity levels may significantly influence product quality and production efficiency. Fiber-optic humidity sensors have some notable advantages over conventional electronic humidity sensors, such as miniature size, light weight, immunity to electromagnetic interference, water and corrosion resistance and radiation tolerance. Several types of fiber optic humidity sensors have been proposed and demonstrated to date, including those based on fluorescence [7], fiber Bragg gratings [8], interferometry [9] and surface plasmon resonance [10]. Recently we proposed and experimentally demonstrated a humidity sensor based on a spherical silica WGM resonator coated with a layer of hygroscopic Agarose layer [11].

Most of the reported sensors have focused on measurements of relatively high levels of humidity, typically above $30 \% \mathrm{RH}$, whereas only a very few studies to date have considered sensing low humidity levels. Measurement of humidity values below $25 \% \mathrm{RH}$ is important in many industrial applications, for example, in monitoring of industrial gases, where the presence of even low levels of water vapor can cause the formation of aggressive chemicals and corrosion of pipes and containers [12]. Alvarez-Herrero et al. proposed a low relative humidity sensor based on a side-polished fiber coupled to a $\mathrm{TiO}_{2}$ waveguide operating at a wavelength $633 \mathrm{~nm}$ with 
sensitivity of up to $0.5 \mathrm{~nm} / \% \mathrm{RH}$ and an estimated resolution of $0.2 \% \mathrm{RH}$ in the range from $0-15 \% \mathrm{RH}$ [13]. Ma et al. [14] proposed and demonstrated a very low humidity $(<10 \%)$ WGM sensor coated with a $\mathrm{SiO}_{2}$ nano-particles, capable of detecting a change of $1 \mathrm{ppm}$ of water molecules but suffering from poor stability of the coating and a significant hysteresis.

In this paper we demonstrate a novel approach to measurements of very low RH values based on the WGMs effect in a small silica microsphere coated with a thin layer of Agarose hydrogel. While measurement of humidity in the higher $\mathrm{RH}$ region (30\% to $70 \% \mathrm{RH}$ ) using an Agarose-coated microsphere was previously demonstrated in our earlier work [11], the approach proposed here utilizes a significantly different interrogation method overcoming the limitation of the optical spectrum analyser's (OSA) resolution. This study aims to develop deeper understanding of the influence of different low relative humidity levels on the spectral positions of the WGM resonances and their spectral shifts. The experimental demonstration of the sensor's capability to measure very low concentrations of water in air and the results of studies of stability, repeatability, hysteresis, response time and temperature dependence make the sensor's performance well suited for measurements of low levels of relative humidity.

\section{Sensing PRINCIPLE}

WGMs in a microsphere can be characterized by a set of integers: $n, l$, and $m$, which represent the radial, azimuthal, and polar mode numbers, respectively. When $l=m$ and, $n=1$, the mode is referred to as a fundamental. A WGM can have either a transverse electric (TE) or transverse magnetic (TM) polarization, which can be selectively excited by controlling the polarization of the coupled light. For simplicity, we only consider the TE polarization. Using a ray optics interpretation, when the light is trapped inside the microsphere, the condition for a WGM resonance is

$$
2 \pi n_{e f f} R=l \lambda_{R}
$$

where $\lambda_{R}$ is the resonance wavelength, $n_{\text {eff }}$ is the effective refractive index of the microsphere and $R$ is its radius. Any fractional change in either refractive index or radius will result in a shift in the WGM resonance wavelength. This shift can be expressed as

$$
\frac{\Delta \lambda_{R}}{\lambda_{R}}=\frac{\Delta n_{\text {eff }}}{n_{e f f}}+\frac{\Delta R}{R}
$$

Teraoka et al. studied the properties of spherical microresonators coated with high RI materials and demonstrated that a high RI coating layer enhances the sensitivity of the WGM wavelength shift to both surrounding RI and the resonator's radius. They also described a perturbation theory for the wavelength shift of WGM resonances in a microsphere coated with a high-RI layer [15-17]. Later, Nai Lin et al. used this approximate model to calculate the thickness-dependent sensitivity of a zeolite coated microsphere to ammonia gas [18]. They demonstrated that the zeolite coating enhances the typically small microsphere surface-to-mass ratio and shows good sensitivity to ammonia. In our studies, we used the same model to determine the resonance conditions of the Agarose coated microsphere. Fig. 1 shows a schematic diagram of the sphere of radius $a_{0}$ with an Agarose coating layer of thickness $t$. The RIs of the microsphere and the Agarose coating are $n_{l}$ and $n_{2}$ and the RI of the surrounding medium is $n_{3}$.

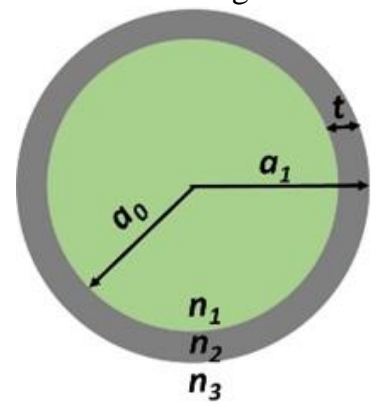

Fig. 1. Schematic diagram of the Agarose coated microsphere.

The characteristic equation for the resonant wavelength $\lambda_{R}$ of the WGM with the mode number $l$ is given by [17]:

$$
\eta_{0} \frac{\chi_{l}^{\prime}\left(n_{3} k a_{1}\right)}{\chi_{l}\left(n_{3} k a_{1}\right)}=\frac{B_{l} \psi_{l}^{\prime}\left(n_{2} k a_{1}\right)+\chi_{l}^{\prime}\left(n_{2} k a_{1}\right)}{B_{l} \psi_{l}\left(n_{2} k a_{1}\right)+\chi_{l}\left(n_{2} k a_{1}\right)}
$$

where

$$
B_{l}=\frac{\eta_{1} \psi_{l}^{\prime}\left(n_{1} k a_{0}\right) \chi_{l}\left(n_{2} k a_{0}\right)-\psi_{l}\left(n_{1} k a_{0}\right) \chi_{l}^{\prime}\left(n_{2} k a_{0}\right)}{\psi_{l}^{\prime}\left(n_{2} k a_{0}\right) \psi_{l}\left(n_{1} k a_{0}\right)-\eta_{1} \psi_{l}\left(n_{2} k a_{0}\right) \psi_{l}^{\prime}\left(n_{1} k a_{0}\right)}
$$

where $\eta_{1}=\frac{n_{1}}{n_{2}}$, for TE mode, $k=2 \pi / \lambda_{R}$ is the wave number for $1^{\text {st }}$ order radial WGM, $a_{l}=a_{0}+t$ is the total radius of the microsphere including the Agarose layer. $\psi_{l}$ and $\chi_{l}$ are the $l_{t h}$ orders of spherical Ricatti-Bessel and spherical RicattiNeumann functions, respectively. Each value of $l$ represents multiple values of $\lambda_{R}$ that satisfy the characteristic equation. The electric field distribution of the TE WGMs in the coated microsphere can be described as [17]:

$E_{l}= \begin{cases}A_{l} \psi_{l}\left(n_{1} k r\right) & r<a_{0} \\ B_{1 l} \psi_{l}\left(n_{2} k r\right)+B_{2 l} \chi_{l}\left(n_{2} k r\right) & a_{0}<r<a_{1}(4) \\ C_{l} \chi_{l}\left(n_{3} k r\right) & r>a_{1}\end{cases}$

where $\mathrm{A}_{l}, B_{l}=\frac{B_{1 l}}{B_{2 l}}$ and $\mathrm{C}_{l}$ are constants and can be determined by matching the boundary conditions for a specific azimuthal mode number $l$.

\section{EXPERIMENTS AND DISCUSSION}

\section{A. Experimental investigation of the humidity response}

A schematic representation of the proposed humidity sensor and its characterization setup are shown in Fig. 2.

First, an adiabatic tapered fiber was fabricated from a standard SMF-28 single mode fiber by using a customized micro heater brushing technique [19]. In our experiment the 
tapered waist diameter is approximately $3 \sim 4$ microns.

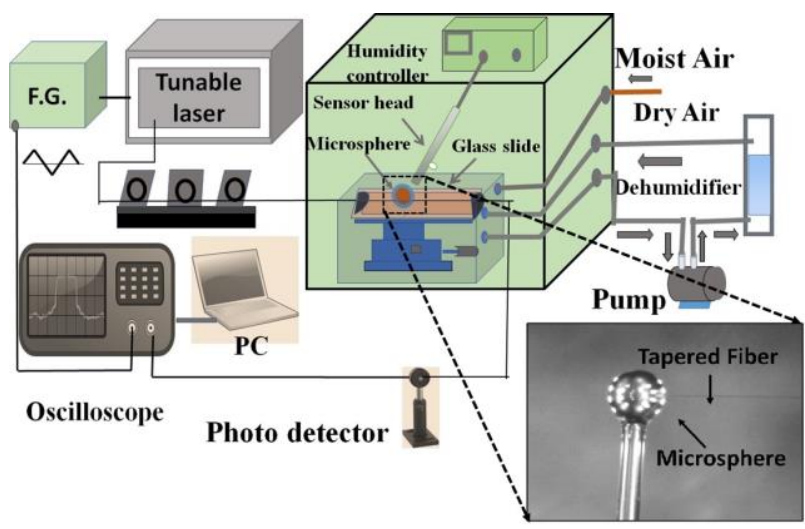

Fig. 2. Schematic representation of the experimental setup for humidity measurements. The dotted-line box indicates the location of the microsphere and coupling taper, shown in the inset image

To improve mechanical stability, the fabricated fiber taper was fixed on a glass slide at a height of $\sim 5 \mathrm{~mm}$ from the slide surface using two drops of UV curable epoxy (Norrland). The microsphere for our experiments was fabricated at the tip of a short fiber section (also an SMF-28 fiber). The cleaved end of the fiber was placed inside a fusion splicer (Sumitomo, Type36). A series of electric arc discharges were applied to the cleaved end of the fiber, so that its tip was gradually softened and became spherical in shape due to surface tension. The diameter of the microsphere measured with an optical microscope was $162 \pm 0.15 \mu \mathrm{m}$. Agarose hydrogel was prepared using white Agarose powder from Sigma Aldrich (A6013) by adding 2.25\% wt./vol. of the Agarose powder into deionized (DI) water followed by stirring at $80{ }^{\circ} \mathrm{C}$ temperature until the powder completely dissolved in the DI water. The refractive index of the Agarose gel was measured as 1.3385 with the help of Abbe refractometer. The microsphere sample was then mounted on a computer- controlled translation stage. The pulling speed of the microsphere from the hot Agarose gel was set to $1 \mathrm{~mm} / \mathrm{sec}$. The coated microsphere was kept at room temperature for one day before use to allow for drying. For the humidity experiments, the sphere was mounted on an $x-y-z$ translational stage to bring its equator region into direct contact with the tapered fiber to excite WGMs, as shown in Fig. 2. The gap between the fiber taper and microsphere may significantly influence the coupling efficiency of the sensor. To ensure the most efficient and stable coupling for our experiment, direct contact between the tapered fiber and the microsphere surface was used to ensure stable coupling conditions.

The characterization of the WGM transmission spectrum of the fiber taper was performed with the help of a tunable external cavity laser (Tunics Plus) with a linewidth of $150 \mathrm{kHz}$ and operating wavelength range of 1490-1640 nm. The wavelength of the tunable laser was swept finely and continuously within $3 \mathrm{GHz}$ around the selected WGM resonance. A sawtooth current modulation realized by the function generator produced repeated sweeps across the spectral width of the WGM resonance. The output end of the tapered fiber was connected to a photo detector. The transmitted optical power was monitored and measured in real time with a high-speed oscilloscope connected to the photodetector, and the transmitted power data were recorded as a function of time using a customized LabVIEW program.

The polarization controller was adjusted manually to achieve maximum light coupling efficiency. Fig. 3 (a) illustrates the WGM transmission spectrum of the uncoated microresonator and a Lorentzian fitting of the spectral dip used to estimate the value of its Q-factor. The Q-factor of the WGM resonance is calculated as $1.38 \times 10^{7}$ using the formula $Q=\lambda_{R} / \Delta \lambda_{F W H M}$, where $\lambda_{R}$ is the resonance wavelength and $\Delta \lambda_{F W H M}$ is the full width at half-maximum of the Lorentz function.
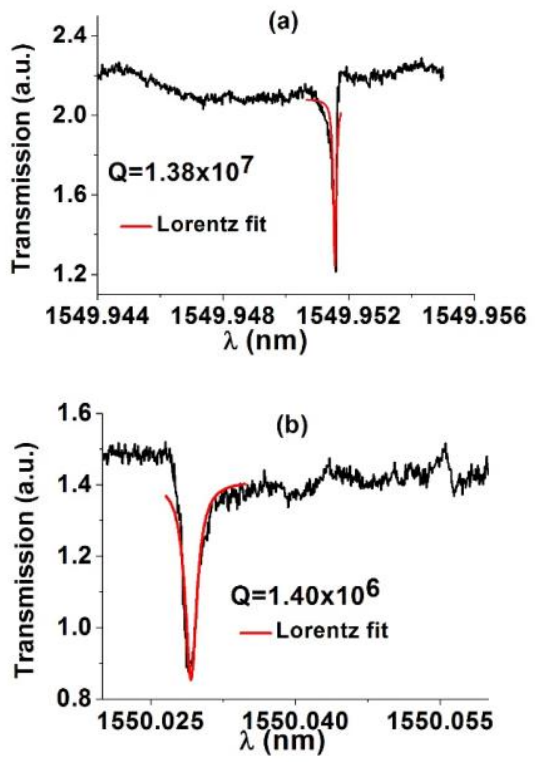

Fig. 3. Q factor estimate for the $162 \mu \mathrm{m}$ diameter silica sphere (a) before and (b) after its coating with Agarose.

After application of the Agarose coating, the Q-factor of the microsphere decreases to $1.40 \times 10^{6}$ as a result of the increase in loss due to absorption and surface scattering of the coating layer. This result is consistent with that reported in our recent study of the Q-factor dependence on the Agarose coating thickness [11]. Accurate control of the Agarose coating thickness plays an important role in fabrication of the proposed humidity sensor. The sensitivity to humidity increases with the increase of the coating thickness eventually saturating beyond the threshold value, determined by the parameters of the microsphere. On the other hand, the quality factor of the microsphere decreases with the increase of the coating thickness. This therefore leads to the need to ensure an optimal trade of between the quality factor and the sensitivity of such type of a sensor in real world applications. In addition to the development of the technique for the Agarose thickness control, several other issues, such as sensor packaging and simple interrogation, need to be addressed for the development of practical devices. The corresponding studies are currently on-going. Since the dip coating technique used to apply the 
Agarose hydrogel on the surface of the microsphere does not allow for an accurate control over the layer thickness, we estimated the layer thickness by means of a comparative experiment as follows. A single mode fiber was coated with an Agarose layer using the same hydrogel solution and the same technique as applied previously to the microsphere. The coated fiber then was cleaved to improve the visibility of the coating layer and an SEM image of the cross-section was taken. Fig. 4 illustrates an SEM image of the cleaved fiber end showing an estimated Agarose layer thickness of $3.55 \mu \mathrm{m}$.

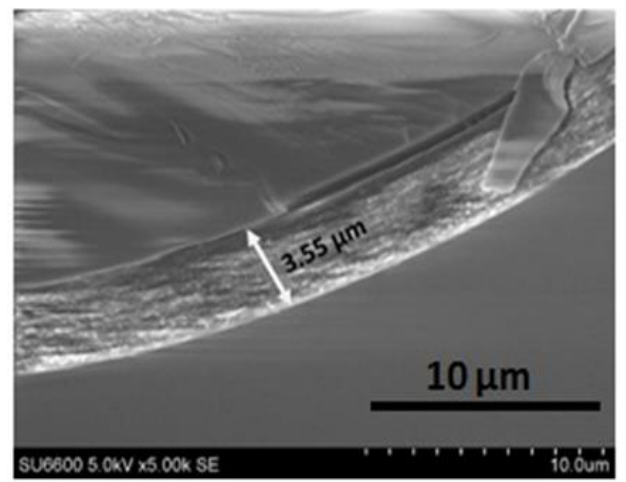

Fig. 4. SEM image of the Agarose coated-fiber cross section.

To investigate the humidity response of the Agarose-coated microsphere resonator, the sensor was placed inside a temperature controlled humidity chamber (ETS5503) with the total volume of 106 liters, shown in Fig. 2. The accuracy of the humidity setting within this chamber is $\pm 2 \% \mathrm{RH}$, which was inadequate for studies in a lower humidity range. To overcome this, the sensor sample together with the coupling taper and the humidity controller was placed inside a smaller (5-liter in volume) enclosure which in turn was placed inside the larger humidity chamber as shown in Fig. 2. Reducing the effective chamber volume allowed the use of a dedicated humidity controller to achieve much better setting accuracy. Temperature control was realized by means of the larger chamber. The RH values inside the small chamber were controlled by a calibrated humidity and temperature sensor controller (ETS 520) with a resolution $\pm 0.1 \% \mathrm{RH}$. Due to the reduced chamber volume, it was possible to accurately characterize the sensor's response in the relative humidity range between $\sim 1$ and $25 \% \mathrm{RH}$.

Fig. 5 shows a comparison between the experimentally measured humidity responses of the WGM resonance for the same microsphere before and after it was coated with an Agarose layer. The proposed sensor's estimated sensitivity is $0.71 \mathrm{pm} / \% \mathrm{RH}$ in the humidity range $1-25 \% \mathrm{RH}$ at the temperature of $17 \pm 0.2^{\circ} \mathrm{C}$. Any increase in humidity inside the chamber gives rise to the adsorption of water molecules on the surface of the microsphere. Physically some of the water molecules replace the air inside the micropores of the coating layer due to the hygroscopic nature of the material and capillary forces, which in turn gives rise to an increase in the effective refractive index of the Agarose layer. The inset of Fig. 5 illustrates measured transmission spectra of the micro- resonator coated with Agarose corresponding to the increasing $\mathrm{RH}$ values. As can be seen from the graph, an increase in RH inside the chamber from $1 \%$ to $25 \%$ resulted in a redshift of the WGMs by $16.6 \mathrm{pm}$. The linear regression value of $\mathrm{R}^{2}=$ 0.99902 demonstrates the high linearity of the sensor's response within $1-25 \% \mathrm{RH}$ range.

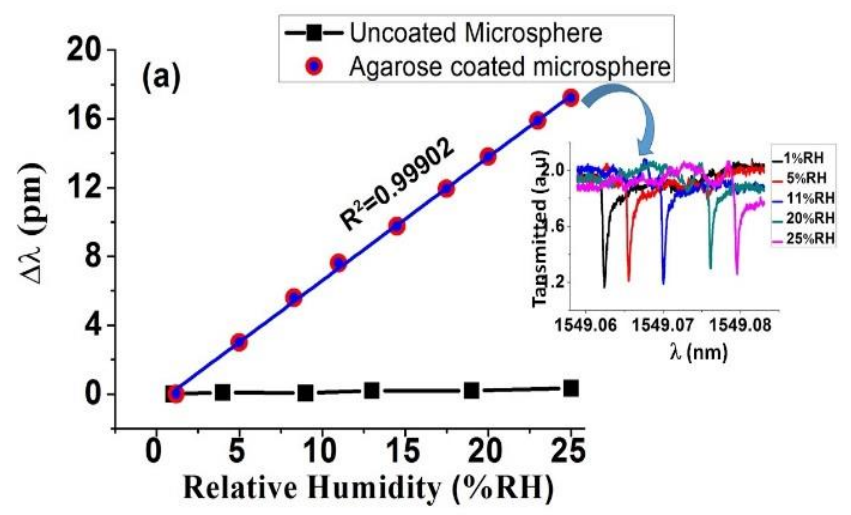

Fig. 5. Wavelength shift of the WGM resonance versus $\mathrm{RH}$ for the microresonator before and after its coating with Agarose. Inset graph illustrates the experimental transmission spectra of the coated micro-resonator corresponding to different $\mathrm{RH}$ values.

It should be noted that although the measurable RH range for the proposed sensor is relatively narrow, since it is limited by the maximum detuning range $(3 \mathrm{GHz})$ of the laser source used in the experiment, studies of the sensor performance at higher RH values confirm that its sensitivity is almost constant $(\sim 0.71 \mathrm{pm} / \% \mathrm{RH})$ in a much broader humidity range of up to $78 \% \mathrm{RH}$. Beyond $80 \% \mathrm{RH}$ its sensitivity increases to 1.32 $\mathrm{pm} / \% \mathrm{RH}$, possibly due to accumulation of water molecules on the surface of the coating, leading to the non-linear changes of the refractive index.

The simulated radial field distribution for the fundamental WGM of the Agarose coated sphere is shown in Fig. 6 (a). From (1) and (2) it was determined that the nearest possible solution for the fundamental resonant mode corresponding to the experimental value is $l=463$ and the corresponding WGM resonance wavelength $\lambda_{R}=1549.966 \mathrm{~nm}$. The parameters used in the numerical simulation are as follows: microsphere radius $a_{0}=81.0 \mu \mathrm{m}$, RI of silica $n_{1}=1.447$, RI of the Agarose coating $n_{2}=1.3385$, RI of the surrounding air $n_{3}=1.0$ and Agarose layer thickness $t=3.55 \mu \mathrm{m}$. Using the method described in [17] we estimated that under the above conditions, approximately a $4.37 \%$ fraction of the light energy is distributed inside the Agarose coating layer. The experimental value of the spectral shift due to humidity was correlated to the numerically calculated change in the RI of the coating without the change in the azimuthal mode number l. Using the model described earlier, we obtained an estimate of the total change in the Agarose layer RI $\Delta n_{\text {Agarose }}=4.7 \times 10^{-4}$ RIU within the studied humidity range. The plot in Fig. 6 (b) shows the calculated data for the Agarose layer RI versus relative humidity. 

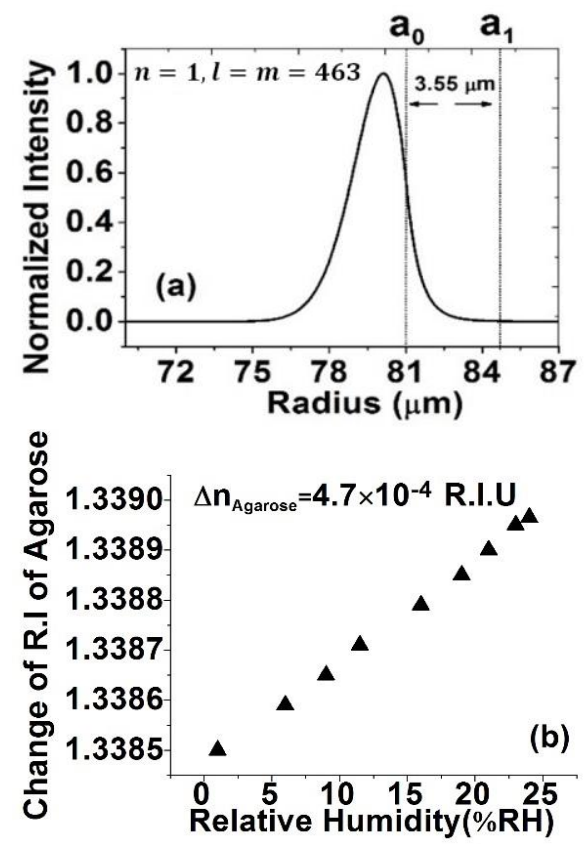

Fig. 6 (a) Simulated electric field intensity distributions for the fundamental WGM; (b) change of the Agarose layer RI corresponding to the RH changes (calculated using the theoretical model) with $n=1, l=m=463$ with $a_{0}=81.0 \mu \mathrm{m}$ and Agarose layer thickness $t=3.55 \mu \mathrm{m}$.

The detection limit (DL) for a sensor represents the smallest measurable physical parameter change and it can be expressed as $\mathrm{DL}=R / S$, where $R$ is the resolution of the sensor and $S$ is its sensitivity. $R$ can be calculated as

$$
R=3 \times \sqrt{\sigma_{N}^{2}+\sigma_{T}^{2}+\sigma_{S R}^{2}}
$$

where $\sigma_{N}, \sigma_{T}, \sigma_{S R}$ represent the standard deviations associated with amplitude noise, temperature and detector spectral resolution respectively [20]. We assumed the signal-to-noise ratio of the system is approximately $60 \mathrm{~dB}$, so that $\sigma_{N}$ is calculated as $10.47 \mathrm{fm}$. The standard deviation due to temperature stabilization is taken as $\sigma_{T}=10 \mathrm{fm}$ [20]. The standard deviation associated with the spectral resolution of the tunable laser whose linewidth of $150 \mathrm{kHz}$ at $1550 \mathrm{~nm}$ $\sigma_{S R}=1.2 \mathrm{fm}$ [20]. The overall sensor resolution is then calculated as $R=43.58 \mathrm{fm}$. Hence, the DL for the microsphere with a $3.55 \mu \mathrm{m}$ thick Agarose layer is calculated as $6.13 \times 10^{-2} \% \mathrm{RH}$ within the $1-25 \% \mathrm{RH}$ humidity range. We estimated the concentration of water molecules inside the 5liter volume chamber as $190.47 \mathrm{ppm}$ at $1 \% \mathrm{RH}$ [21]. Hence, the minimum detection limit of water molecules is estimated as $11.7 \mathrm{ppm}$.

\section{B. Stability tests}

To test the longer-term stability of the sensor's performance, RH measurements were carried out using the same sensor sample after a time interval of one week followed by two more weeks from the initial tests in the same $\mathrm{RH}$ range from $1 \%$ to $25 \%$ at a constant temperature of $17 \pm 0.2{ }^{\circ} \mathrm{C}$.

It can be seen from Fig. 7 (a), that the performance of the sensor is very stable over the two-week period with less than $1 \%$ fluctuations from the initial test values, the sensor was tested for repeatability by continuously increasing and decreasing relative humidity inside the chamber from $\sim 12$ to $25 \%$ RH for several times. Fig. 7 (b) illustrates a series of tests where the sensor was exposed to continuously increasing and decreasing relative humidity levels from 12 to $25 \% \mathrm{RH}$ inside the chamber and the estimated response and recovery times for the sensor. The increase of humidity in the experiment was achieved by natural means whereas the dehumidification was controlled by pumping dry air inside the chamber, so that the latter process took less time within each of the test cycles. As one can see from the figure, the sensor demonstrates good repeatability and a short recovery time in the order of seconds. It should be noted that the response and recovery times include those of the chamber itself and given the volume of the chamber it is likely that the actual response and recovery times of the sensor are much shorter.

Results of studies of the sensor's hysteresis are presented in Fig. 7 (c) and 7 (d). It is clear from the graph that the proposed sensor has a very small hysteresis effect even with a relatively thick $3.55 \mu \mathrm{m}$ Agarose coating. The error due to hysteresis is found to be less than $2 \%$ (Fig. 7 (d)). This is likely due to the difference between the rates of adsorption and desorption of water molecules at the surface of the Agarose layer. This also leads to slight deviation form linearity of the sensor's response observed in Fig. 7 (d). It is clear from the graph that the proposed sensor has very small hysteresis. It should be noted that both response and relaxation times and hysteresis properties for the proposed sensor are significantly better than those reported in $[14,22]$ for a WGM microsphere resonator in a low humidity range.

\section{Response time}

The sensor's response time was evaluated in detail. The response time is defined as the time it takes for the wavelength shift to reach $90 \%$ of its maximum value when the $\mathrm{RH}$ is increased from $0 \%$ to a target $\mathrm{RH}$ value. The temperature of both chambers was maintained constant and monitored by the ETS 520 controller.

Several experimental techniques have been proposed to determine the response time of humidity sensors, with the most popular but simple method based on monitoring of a rapid human breathing cycle [23-24]. Since our experiments concerned the sensor's performance in the low RH range, this technique was not applicable in our case, since human breath is associated with much higher than $25 \% \mathrm{RH}$ levels of humidity.

The response time calculated from the experiments carried out in the humidity chamber was approximately 3.7 second as shown in Fig. 7 (b). It should be noted however that our humidity controlled chamber also is not ideal for studying the response time of the sensor because the flows of the humid/dry air in and out of the chamber are very slow.

Therefore, in this study we used the following simple method to estimate the response time of the sensor. The difference between the RH settings in both the large and small 
chambers was maintained constant at 5\% RH. The sensor's response was monitored continuously while the cover of the small chamber was rapidly removed allowing the RH levels of both small and big chambers to equalize. The results of this experiment are presented in Fig. 8.
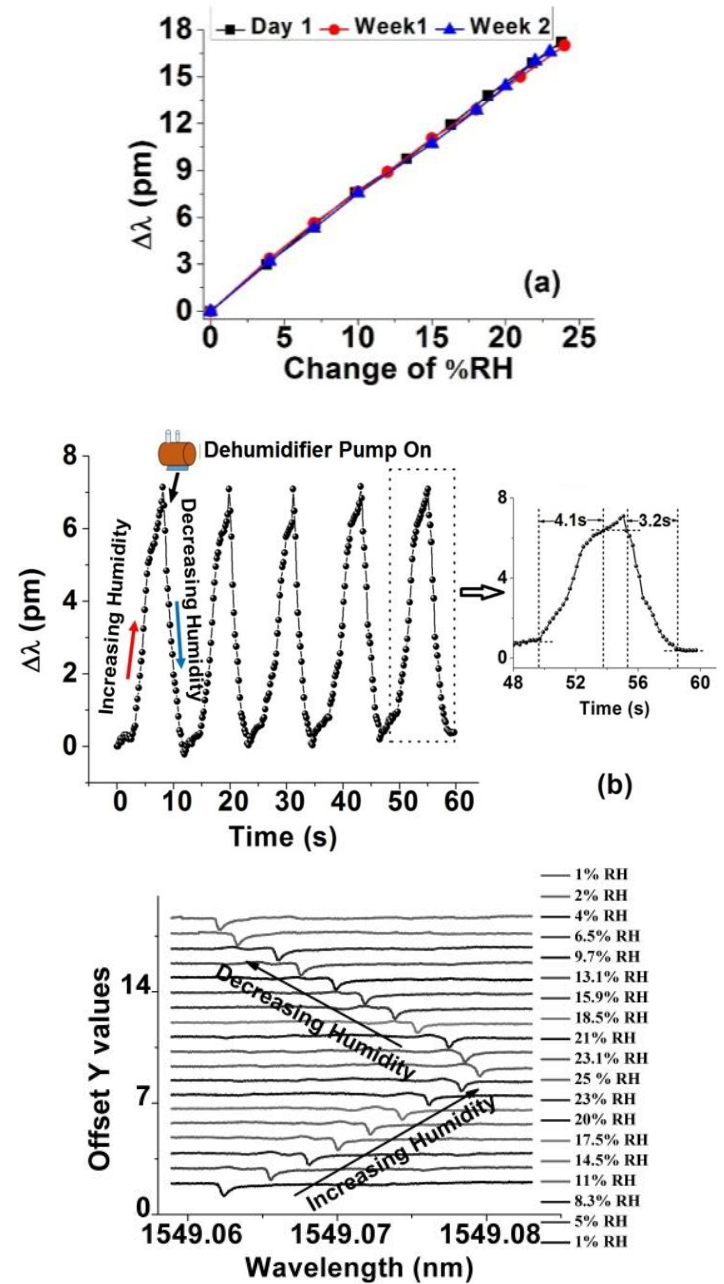

(c)

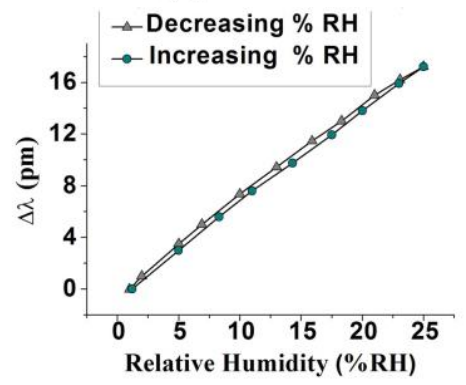

(d)

Fig. 7. (a) RH responses of the sensor taken within 2 weeks; (b) Studies of repeatability and estimates for response and recovery times; (c) WGM spectra at different RH levels at increasing and decreasing humidity at $17^{\circ} \mathrm{C}$; (d) WGM resonance shift versus $\mathrm{RH}$ at $17^{\circ} \mathrm{C}$.

The sensor's temporal response was plotted by processing a series of digitized oscilloscope images (1000 data points per frame) using a PC to determine the position of the WGM dip corresponding to each of the snapshot images. The frequency of the snapshots was limited to $\sim 5$ frames/sec by the speed of transfer and processing of each data set. The estimated response time of the Agarose-coated $162 \pm 0.15 \mu \mathrm{m}$ diameter microsphere from Fig. 8 was calculated as $0.9 \mathrm{~s}$.

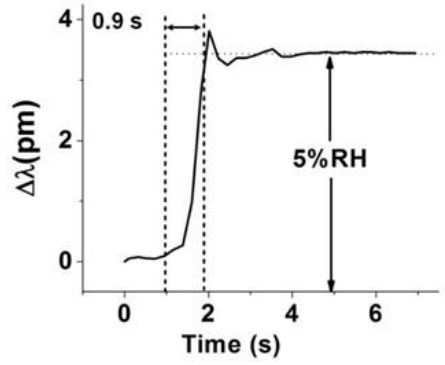

Fig. 8. Temporal response of the sensor.

\section{Temperature effects}

The influence of temperature on the $\mathrm{RH}$ sensor performance was investigated in the range from $20^{\circ} \mathrm{C}$ to $36^{\circ} \mathrm{C}$ at three different humidity levels, i.e. $10 \% \mathrm{RH}, 15 \% \mathrm{RH}$ and $25 \% \mathrm{RH}$, set inside the smaller chamber. The lower temperature value of the studied range is determined by the room temperature since the humidity chamber does not have a means of cooling, while the upper temperature limit of $36^{\circ} \mathrm{C}$ is determined by the gelling temperature of Agarose $\left(36^{\circ} \mathrm{C}-42^{\circ} \mathrm{C}\right)$. As the temperature of the larger chamber was gradually raised from $20^{\circ} \mathrm{C}$ to $36^{\circ} \mathrm{C}$ in small steps, the wavelength shift of the WGM spectrum was recorded using a high speed digital oscilloscope connected to a PC. The overall temperature response for the Agarose coated microsphere is shown in Fig. 9 (a). The estimated temperature sensitivity values calculated from the Fig. 9 (a) are $2.3 \mathrm{pm} /{ }^{\circ} \mathrm{C}$ at $25 \% \mathrm{RH}, 2.25 \mathrm{pm} /{ }^{\circ} \mathrm{C}$ at $15 \% \mathrm{RH}$ and $2.5 \mathrm{pm} /{ }^{\circ} \mathrm{C}$ at $10 \% \mathrm{RH}$. We estimated the linearity error of temperature was approximately $3.5 \%$.

The temperature sensitivities at higher RH levels (15 and $25 \%$ ) are very similar; but the sensor displays slightly higher temperature response at $10 \% \mathrm{RH}$. One possible explanation for the observed higher temperature sensitivity at $10 \% \mathrm{RH}$ humidity is that because at lower levels of humidity the pores of the Agarose layer are mostly filled with air, it results in a greater refractive index contrast between the Agarose layer and air, and thus greater sensitivity to any changes in the surrounding refractive index [25]. While the cross-sensitivity of the proposed sensor to temperature at low humidity is quite high it should be noted that our proposed sensor has several orders of magnitude smaller temperature cross-sensitivity compared with some other types of fiber optic humidity sensors, such as long period gratings [26] or fiber Bragg grating-based humidity sensors [27].

Fig. 9 (b) illustrates the temperature increasing and decreasing responses of the same sensor sample at $15 \% \mathrm{RH}$. It is found the responses are in a good agreement with each other with negligible hysteresis.

\section{CONCLUSION}

A novel approach to measure ultra-low concentrations of water vapor, corresponding to low $\mathrm{RH}$ values, using an Agarose coated microsphere resonator based on the WGMs 


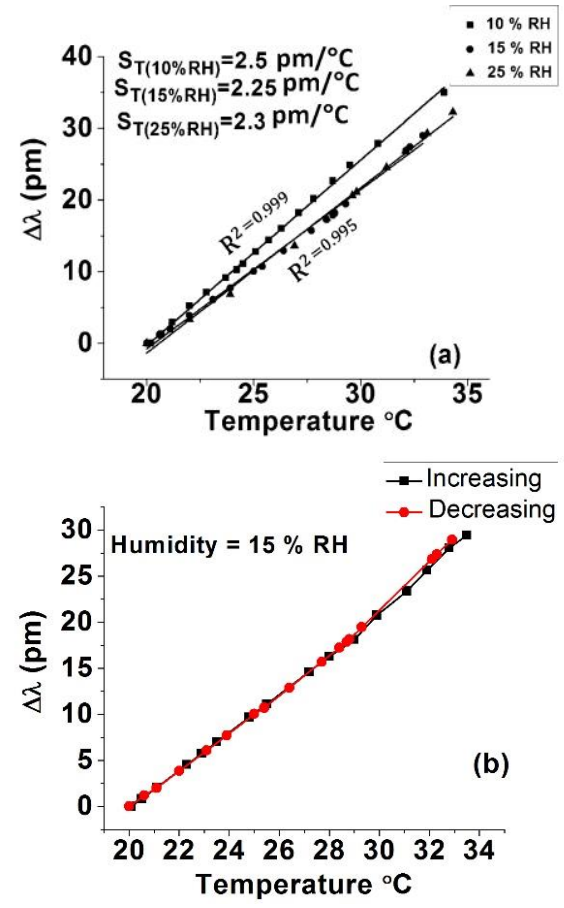

Fig. 9. Resonant wavelength shift versus temperature for the $162 \pm 0.15 \mu \mathrm{m}$ microsphere coated with $2.25 \%$ wt./vol. Agarose solution: (a) $10 \%$ RH, $15 \%$ $\mathrm{RH}$ and $25 \% \mathrm{RH}$; (b) Hysteresis of temperature response at $15 \% \mathrm{RH}$.

effect has been proposed and experimentally demonstrated in the humidity range from 1 to $25 \% \mathrm{RH}$. The WGMs are excited in a silica microsphere dip-coated with an Agarose gel by evanescent coupling to an adiabatic tapered fiber and observed in the transmission spectrum of the taper. The spectral shift of the WGM resonances is induced by the adsorption or desorption of water molecules at the microsphere surface, resulting in a change of RI of the Agarose coating. The demonstrated experimentally sensitivity of the sensor is 0.71 $\mathrm{pm} / \% \mathrm{RH}$ with an estimated detection limit of $11.7 \pm 0.32 \mathrm{ppm}$ after considering $2 \%$ hysteresis error and $3.5 \%$ linear error of temperature using the present setup, which we believe is the highest DL for water vapor detection reported to date. The proposed sensor is very compact and demonstrates good repeatability and stability. The response time of the sensor is $0.9 \mathrm{~s}$, which is very promising for applications where a fast response is required at low levels of humidity. The cross sensitivity to temperature at different humidity levels is investigated. The sensor's temperature sensitivity is almost constant above $15 \%$ RH. A more detailed study of the proposed sensor is currently ongoing with the aim to improve the sensing time as well as optimize the coating thickness, which determines the sensitivity and dynamic range.

\section{REFERENCES}

[1] N. M. Hanumegowda, C. J. Stica, B. C. Patel, I. White, and X. Fan, "Refractometric sensors based on microsphere resonators," Appl. Phys. Lett., 87, 201107, 2005.

[2] V. S. Ilchenko, P. S. Volikov, V. L. Velichansky, F. Treussart, V. Lefèvre-Seguin, J.-M. Raimond, and S. Haroche, "Strain tunable high-Q optical microsphere resonator," Opt. Commun., 145, pp. 86-90, 1998.

[3] T. Ioppolo, M. Kozhevnikov, M. V. Ötügen, and V. Sheverev, "Performance of a whispering gallery mode resonator-based micro- optical force sensor," Presented at the 45th AIAA Aerospace Sciences Meeting and Exhibition, Reno, Nev., Jan. 2007.

[4] C. Y. Chao, W. Fung, and L. J. Guo, "Polymer microring resonators for biochemical sensing applications," IEEE J. Sel. Top. Quantum Electron., vol. 12, pp. 134-142, 2006.

[5] F. Vollmer, D. Braun, A. Libchaber, M. Khoshsima, I. Teraoka, and S. Arnold, "Protein detection by optical shift of a resonant microcavity," Appl. Phys. Lett., vol. 80, pp. 4057-4059, 2002.

[6] N. Lin, L. Jiang, S. Wang, Q. Chen, H. Xiao, Y. Lu and H. Tsai, "Simulation and optimization of polymer-coated microsphere resonators in chemical vapor sensing", Appl. Opt., vol. 50, pp. 5465-5472, 2011.

[7] H. E. Posch, O. S. Wofbeis, "Optical sensors, 13: fibre-optic humidity sensor based on fluorescence quenching", Sens. Actuator., 15, 77-83, 1998.

[8] F. J. Arregui, I. R. Matias, K. L. Cooper, and R. O. Claus, "Simultaneous measurement of humidity and temperature by combining a reflective intensity-based optical fiber sensor and a fiber Bragg grating”, IEEE Sensors J., vol. 2, pp. 482-487, 2002.

[9] J. M. Corres, I. R. Matias, M. Hernaez, J. Bravo, and F. J. Arregui, "Optical Fiber Humidity Sensors Using Nano structured Coatings of SiO2 Nano particles", IEEE Sensors J., vol. 8, pp. 281-285, 2008.

[10] M. Hernáez, C. R. Zamarreño, I. R. Matías, F. J. Arregui, "Optical fiber humidity sensor based on surface plasmon resonance in the infra-red region", JPCS, vol. 178 012019, 2009.

[11] A. K. Mallik, D. Liu, V. Kavungal, Q. Wu, G. Farrell and Y. Semenova, "Agarose coated spherical micro resonator for humidity measurements", Opt. Express, vol. 24, pp. 21216-21227, 2016.

[12] J. Majewski, "Polymer-based sensors for measurement of low humidity in air and industrial gases", Electrical review, vol. 8, pp. 74-77, 2016.

[13] A. Alvarez- Herrero, H. Guerrero, and D. Levy, "High sensitivity sensor of low relative humidity based on overlay on side-polished fibers", IEEE Sensors J., vol. 4, pp. 52-56, 2004.

[14] Q. Ma, L. Huang, Z. Guo and T. Rossmann, "Spectral shift response of optical whispering-gallery modes due to water vapor adsorption and desorption”, Meas. Sci. Technol., vol. 21115206 (7pp), 2010.

[15] J. C. Knight, G. Cheung, F. Jacques, and T. A. Birks, "Phase-matched excitation of whispering-gallery-mode resonances by a fiber taper", Opt. Lett., vol. 22, pp. 1129-1131, 1997.

[16] I. Teraoka, S. Arnold, and F. Vollmer, "Perturbation approach to resonance shifts of whispering-gallery modes in a dielectric microsphere as a probe of a surrounding medium", J. Opt. Soc. Am. B, vol. 20, pp. 1937-1946, 2003.

[17] I. Teraoka, and S. Arnold, "Enhancing the sensitivity of a whisperinggallery mode microsphere sensor by a high-refractive index layer", $J$. Opt. Soc. Am. B, vol. 23, pp. 1434-1442, 2006.

[18] N. Lin, L. Jiang, S. Wang, L. Yuan, H. Xiao, Y. Lu, and H. Tsai, "Ultrasensitive chemical sensors based on whispering gallery modes in a microsphere coated with zeolite", Appl. Opt., 49, 6463-6471, 2010.

[19] G. Brambilla, V. Finazzi, and D. Richardson, "Ultra-low-loss optical fiber nanotapers," Opt. Express, vol. 12, pp. 2258-2263, 2004.

[20] I. M. White and X. Fan, "On the performance quantification of resonant refractive index sensors", Opt. Express, vol. 16, pp. 1020-1028, 2008.

[21] The Rotronic Humidity Handbook, Rotronic Instrument Corp., www.rotronic-usa.com.

[22] Q. Ma, Huang, Z.L. Guo, T. Rossmann, "Whispering- gallery mode silica micro-sensors for temperature and gas-phase concentration measurements," Presented at the 27th AIAA Aerodyn. Meas. Technol. and Ground Test. Conference, 2010.

[23] J. Mathew, Y. Semenova, G. Farrell, "Experimental demonstration of a high-sensitivity humidity sensor based on an Agarose-coated transmission-type photonic crystal fiber interferometer", Appl. Opt., 52, pp. 3884-3890, 2013.

[24] F. J. Arregui, Z. Ciaurriz, M. Oneca, I. R. Matias, "An experimental study about hydrogels for the fabrication of optical fiber humidity sensors", Sens. and Act. B, vol. 96, pp. 165-172, 2003.

[25] R. Gao, Y. Jiang and W. Ding, "Agarose gel filled temperatureinsensitive photonic crystal fibers humidity sensor based on the tunable coupling ratio," Sens. and Act. B, vol. 195, pp. 313-319, 2014.

[26] L. Wang, Y. Liu, M. Zhang, D. Tu, X. Mao and Y. Liao, "A relative humidity sensor using a hydrogel-coated long period grating," Meas. Sci. Technol., vol. 18, pp. 3131-3134, 2007.

[27] S. F. H. Correia, P. Antunes, E. Pecoraro, P. P. Lima, H. Varum, L. D. Carlos, R. A. S. Ferreira and P. S. André, "Optical fiber relative humidity sensor based on a FBG with a di-ureasil coating," Sensors, vol. 12, pp. 8847-8860, 2012. 